\title{
Prospective et recherche pour les politiques publiques en phase de transition ${ }^{\star}$
}

\author{
Jacques Theys* \\ Politologue, Président de l'association Serge Antoine Paris, France
}

Les réflexions qui suivent s'appuient sur une pratique de longue date à la fois de la prospective, de la recherche, et des politiques publiques ${ }^{1}$. Leur objet est d'aborder ces trois thèmes dans une perspective de transition - et ceci dans la globalité de leurs relations : faire une prospective des besoins de recherche liés aux politiques de transition écologique et énergétique; proposer une réflexion sur la place future des recherches pour les politiques publiques et de la prospective dans les transitions en cours; mais aussi, plus modestement, situer la place future de la prospective dans les recherches en appui des politiques de transition. Mais comme toute bonne prospective doit s'inscrire dans le temps long, elles s'attachent aussi à tirer les leçons de l'expérience passée tant en France que dans quelques pays comparables.

\footnotetext{
* Voir dans ce numéro le texte d'introduction de Marcel Jollivet.

* Auteur correspondant :

jacques . theys@numericable.fr

Considéré comme le véritable initiateur du ministère de l'Environnement - à côté de George Pompidou et de Robert Poujade - Serge Antoine en a été aussi le premier Directeur des Études et de la recherche - de 1977 à 1984, la structure étant alors commune avec l'Équipement. Son directeur-adjoint était Claude Martinand, futur vice-président du Conseil général des Ponts et Chaussées. C'est de cette période que datent - du côté environnement - les premiers programmes incitatifs.

${ }^{1}$ Expérience à la fois en tant que responsable de la prospective au sein du ministère en charge de l'équipement (1993-2007), de celui en charge de l'environnement (2007-2011); qu'animateur de plusieurs programmes de recherche incitative (de 82 à 88 sur les risques, de 89 à 93 sur «Économie et Société face aux changements globaux», de 2007 à 2012 sur les politiques de transport); qu'adjoint au directeur de la recherche au ministère de l'Environnement et que membre de plusieurs cabinets ministériels. Jacques Theys a été, en outre, enseignant à l'École des hautes études en sciences sociales (EHESS).
}

Pour aborder ces différentes questions, l'article sera structuré en trois temps : à un premier temps centré sur la problématique générale de la recherche pour les politiques publiques et sur les leçons de l'expérience, succédera un second s'attachant plutôt aux besoins futurs de recherche (quelles recherches pour les politiques de transition écologique, climatique et énergétique ?); le troisième portera sur la place que la recherche pour les politiques publiques (et, à l'intérieur de celle-ci la prospective) devrait occuper dans la phase actuelle de «Grande transition»-qui n'est pas seulement écologique - que nous traversons. Ce sont à chaque fois des questions très vastes; il ne s'agira naturellement que de lancer quelques idées pour alimenter le débat. S'il ne sera question ici que des recherches qui concernent l'environnement, le propos n'en peut pas moins être généralisé aux autres domaines d'intervention du ministère en charge du développement durable (les transports ou l'urbanisme, par exemple).

\section{La recherche pour les politiques publiques entre science et politique: quelles leçons de l'expérience?}

\begin{abstract}
«Du visible à l'invisible: des politiques de l'environnement de plus en plus déterminées par la science »
\end{abstract}

(Theys et Kalaora, 1992).

Il est intéressant d'observer - mais ce n'est pas une surprise-que les références récentes sur la recherche en appui des politiques publiques, globalement peu nombreuses, portent très fréquemment sur les champs d'action du ministère de l'Environnement et, parmi ceux-ci, plus particulièrement sur celui de l'environnement au sens strict du terme. C'est, en effet, l'une des spécificités majeures des politiques de l'environnement -qu'elles 
partagent avec l'espace ou la mer - de reposer énormément sur les apports de la science. Dans ce domaine, en effet, les sciences ne servent pas seulement à rationaliser les décisions, mais aussi à construire les problématiques, à structurer le débat public, à proposer des solutions, à contribuer à leur mise en œuvre et finalement à évaluer les résultats: elles ont donc vocation à être présentes dans tout le cycle des politiques publiques. Elles sont, en effet, les seules à pouvoir rendre visibles et compréhensibles des phénomènes naturels et des interrelations très complexes qui échappent en grande partie à la perception directe des groupes sociaux et des citoyens et à celle des acteurs publics.

Il faut noter que cette place de la science n'a pas toujours été aussi centrale : il y a un demi-siècle ou plus, c'est, au contraire, à partir des dégradations visibles de l'environnement (les fumées d'usine, l'odeur du soufre, le bruit, la couleur des rivières, la dégradation des paysages, le contact direct avec la nature) que se sont structurés les premiers mouvements environnementalistes ou les premières politiques environnementales. Mais peu à peu les problèmes se sont complexifiés, et l'histoire des politiques de l'environnement de ce dernier demisiècle est placée sous le signe d'une importance croissante de leur dimension scientifique. Et cela sous tous les angles (choix des problématiques, importance de la preuve, usage des prévisions, légitimation des priorités, normalisation, proposition de solutions, évaluation des objectifs, etc.). Il est progressivement devenu de plus en plus difficile pour ces politiques de ne pas s'appuyer aussi sur une forte légitimation scientifique. Il très significatif qu'aux États-Unis, pays qui a inventé la politique de l'environnement, on a commencé par mettre en place une agence pour la protection de l'environnement - qui a pu compter près de 3000 chercheurs. Mais en fait, tous les pays (comme plus tard la communauté internationale) ont dû peu à peu s'organiser pour faire face à ce défi de l'articulation entre connaissances scientifiques et action publique environnementale. Ils l'ont fait en adoptant quatre voies principales :

- une valorisation intelligente du capital de ressources scientifiques existantes: veille et synthèses à destination des responsables publics, appel à l'expertise, colloques, création de conseils scientifiques auprès des gouvernements ou de think-tanks, etc.;

- le développement de capacités internes de recherche ou de connaissances au sein ou à la périphérie des institutions en charge des politiques publiques: recrutement de chercheurs, création d'agences généralistes, de centres techniques ou d'instituts spécialisés, programmes incitatifs de recherche;

- l'auto-organisation, parfois favorisée pas des incitations, de la communauté scientifique: création de programmes, de départements ou d'instituts, pluridisciplinaires ou pas, spécifiquement axés vers l'appui aux politiques publiques ${ }^{2}$;

- et enfin l'influence externe sur les institutions scientifiques: définition d'orientations stratégiques, conventions de recherche, présence dans les conseils d'administration, exercices de prospective scientifique ou technologique, etc.

\section{Encadré. Citons au passage quelques expériences intéressantes.}

Aux Pays-Bas, par exemple - qui ont par ailleurs créé de grands instituts publics à vocation générale, comme le RIVM $^{3}$ (Rijksinstituut voor Volksgezondheid en Milieu) le service de la recherche du ministère s'est, à un certain moment, recentré sur la traduction des connaissances existantes en recommandations pour l'action publique.

En Finlande, le très petit ministère de l'Environnement (à vocation politique) et le très grand Institut de l'environnement (à vocation scientifique) ont mis en place un dispositif très efficace de tutelle et de collaboration permanente - comme c'est aussi le cas en Suède avec un ministère de l'Environnement et de l'Énergie qui soutient et s'appuie sur une grande Fondation pour la recherche stratégique en environnement, MISTRA ${ }^{4}$.

La Grande Bretagne combine avec succès des programmes incitatifs très importants au sein du DEFRA $^{5}$, des instituts universitaires pluridisciplinaires très orientés vers les politiques publiques comme le Tyndall Institute, la meilleure prospective scientifique du monde, et une stratégie très efficace du British Research Council de traduction des résultats de la recherche en recommandations politiques.

En Allemagne, les conseils scientifiques auprès du gouvernement jouent un rôle très important, comme les programmes de recherche menés par l'Agence fédérale de l'environnement.

Au Danemark, le Conseil de la technologie assume une fonction essentielle de «plaque tournante » entre le monde scientifique, le Gouvernement, le Parlement et l'opinion publique. Ces quelques exemples montrent que partout la question des relations science politique s'est imposée comme une question centrale mais qu'elle a pu recevoir des réponses très diverses.

\footnotetext{
${ }^{2}$ Même si l'influence des gouvernements n'y est pas absente on peut mettre le GIEC (Groupe d'experts intergouvernemental sur l'évolution du climat) ou les programmes de l'ANR (Agence nationale de la recherche) dans cette catégorie.

${ }^{3}$ Institut national des Pays Bas sur la santé et l'environnement. ${ }^{4}$ The Swedish Foundation for Strategic Environment Research.

${ }^{5}$ Le DEFRA - ministère de l'Environnement, des Affaires rurales et de l'Alimentation - a été créé après l'affaire de la vache folle.
} 


\section{Les leçons de l'expérience: échec de la séparation, difficultés de la co-construction}

L'une des premières leçons que l'on peut tirer de la diversité de ces expériences est que celles reposant sur la séparation entre le monde de la recherche et celui de l'action publique ont conduit à des résultats médiocres - sans compter un grand gaspillage de ressources et de savoirs. On pourrait citer de multiples exemples de politiques publiques qui se sont trouvées confrontées à de graves échecs pour avoir soit ignoré la diversité des connaissances scientifiques existantes, soit cherché à les instrumentaliser de manière trop réductionniste ou technocratique. Pensons aux échecs sur la taxe carbone ou aux blocages sur les grands projets d'infrastructure. Amy Dahan et Hélène Guillemot ont également bien montré récemment, à propos de l'effet de serre, que le «modèle positiviste»- ou «linéaire»- de relation science-politique (qui conduit d'abord à produire de la science, puis à attendre qu'elle soit traduite en action politique) ne fonctionne, lui non plus, pas bien (Dahan et Guillemot, 2015). On ne peut s'attendre spontanément à ce que les seules compétences internes d'un ministère ou de ses agences techniques suffisent à mener des politiques efficaces, ni à ce que l'offre de recherche fondamentale réponde à la demande politique. Plusieurs exercices successifs de prospective scientifique ${ }^{6}$ ont d'ailleurs bien montré qu'il y avait un grand décalage entre l'offre et la demande de recherche pour les politiques publiques, avec beaucoup de thèmes qui restaient «orphelins», sans réponse scientifique. On ne peut non plus déplorer qu'il existe un grand décalage entre les alertes scientifiques et la décision publique sans se demander dans quelle condition se fait la traduction des unes vers l'autre. Il faut donc se convaincre qu'il y a un problème majeur d'articulation entre eux, un «chaînon manquant » à trouver.

Ce constat, le philosophe allemand Habermas (1973) l'avait très bien anticipé il y a juste un demi-siècle, en expliquant que le mouvement, pour lui irréversible, de «scientifisation» de la politique ne pouvait trouver de solution satisfaisante que dans un effort de coconstruction à la fois des problématiques scientifiques et de l'action publique, impliquant aussi la société; ce qu'il appelait «le modèle pragmatique de décision». Il faut insister sur le fait qu'il ne s'agit pas seulement dans cette perspective de trouver un accord collectif sur des recherches à conduire, mais d'accepter que les scientifiques et la société soient impliqués dans la construction des politiques publiques; et que les acteurs

\footnotetext{
${ }^{6}$ Notamment l'exercice lancé en 1996 sur «La demande de recherche en matière d'environnement» (ministère de l'Environnement) et AGORA 2020, lancé en 2004 (Bain et al., 2008).
}

publics ou la société interviennent dans celle des problématiques scientifiques. Ce qui était pour Habermas une préconisation générale vaut a fortiori encore plus pour l'environnement qui, comme on le sait, a été et reste une construction à la fois scientifique et sociopolitique (que l'on pense à la notion de transition écologique elle-même). Voilà qui nous rapproche un peu plus du rôle de la recherche incitative menée depuis les années soixante-dix par les différents ministères en charge de l'environnement'.

Malheureusement - au moins en France- cette coconstruction ne va absolument pas de soi. Car non seulement les mondes et les temporalités de la recherche et ceux des politiques publiques sont dans la réalité très séparés, mais cette séparation est constitutive d'une part des deux cultures : la culture politico-administrative et la culture scientifique. Elle suppose que les acteurs opérationnels acceptent de reconnaitre qu'ils ne sont pas omniscients et que la recherche peut être une source utile de créativité, de visibilité, d'expertises nouvelles, mais aussi d'évaluations critiques et de solutions efficaces. Et inversement, que les chercheurs considèrent que répondre à des questions qui leurs sont posées par des acteurs publics ou sociaux n'est pas perdre à la fois leur indépendance et leur crédibilité scientifiques, mais au contraire s'ouvrir des chemins nouveaux tout en contribuant au bien commun - même si cela doit les conduire à accepter les risques du compromis et de l'interdisciplinarité Elle suppose aussi qu'il existe des procédures facilitant un dialogue exempt de domination entre chercheurs et utilisateurs - et des traducteurs permettant de passer des questions à la recherche en questions de recherche et des résultats scientifiques en recommandations d'action publique. Elle suppose enfin que la spécificité de cette recherche pour les politiques publiques soit reconnue par les structures institutionnelles de la recherche. Cela fait beaucoup de conditions qui, dans le contexte français, semblent malheureusement de plus en plus difficiles à satisfaire.

\section{L'expérience réussie des programmes incitatifs menés sous l'égide du ministère de l'Environnement et du Développement durable et le paradoxe de leur disparition}

L'expérience menée à partir de la fin des années 1970 au sein du ministère de l'Environnement à travers les programmes incitatifs a cependant montré que ces obstacles pouvaient être en partie dépassés et que cette

\footnotetext{
${ }^{7}$ Sur cette co-construction des sciences et des politiques de l'environnement depuis les années 1960 voir, notamment: Callon et Rip (1992), Godard (2008), Charvolin (2005), Theys (2010), ainsi que les travaux de B. Latour.
} 
co-construction pouvait fonctionner non seulement au profit des politiques publiques mais aussi - et c'est peutêtre le plus intéressant - en donnant naissance à des formes de recherche et de connaissance tout à fait spécifiques et originales: à la fois thématiquement et conceptuellement innovantes, plus anticipatrices, plus multidisciplinaires, plus proches des réalités sociales ou de terrains, mieux appropriables par les acteurs concernés ou dans le débat public; et finalement, pour tout cela, plus conformes aux exigences du développement durable ${ }^{8}$. Fréquemment menés en partenariat, ces programmes ont souvent permis en même temps - il faut le souligner - de constituer des communautés pérennes de chercheur sur des thèmes innovants et de mettre sur l'agenda public de nouvelles préoccupations en servant de terrain d'expérimentation pour leur mise en œuvre concrète. On pourrait ainsi citer de multiples exemples - de la gestion des risques à l'adaptation du littoral au changement climatique en passant par l'écologie appliquée et beaucoup d'autres -, où ils ont permis cette co-construction indispensable à l'émergence d'enjeux précurseurs $^{9}$.

Tout le monde malheureusement n'a pas été convaincu que ce type de programmes et de dialogue - dont les moyens sont toujours restés en France très contingentés - pouvait répondre aux attentes ou exigences conjointes des acteurs publics ou sociaux et de la communauté scientifique. Même si la loi de 2006 sur la recherche a reconnu l'existence des « recherches pour la société et en appui des politiques publiques» comme «troisième pilier de la recherche»-à côté de la recherche fondamentale et de la recherche industrielle ou appliquée - le message a été interprété comme l'appel à en cantonner le développement dans les institutions de recherche et au sein de l'ANR. Les apports de la recherche incitative n'ont pas été estimés à leur juste mesure et chacun est finalement retourné dans son monde. On se retrouve ainsi aujourd'hui devant la situation paradoxale où, au moment même où les perspectives de transition écologique et énergétique

\footnotetext{
${ }^{8}$ Voir les deux rapports réalisés pour le MEDDE : «Expertise sur les formes d'ouverture entre disciplines et de partenariat en recherche dans les programmes de recherche du MEDDE» produit par l'association NSS-Dialogues en 2014 (www.nssdialogues.fr) et «Étude de caractérisation de la recherche finalisée en appui aux politiques publiques mise en œuvre dans les programmes incitatifs de la recherche de la direction de la recherche et de l'innovation du MEDDE » réalisé par AScA en 2015 (http://www.asca-net.com). $C f$. les deux synthèses de ces travaux ci-dessus.

${ }^{9}$ Outre les sources mentionnées dans la note ${ }^{5}$, voir l'interview de Jacques Theys dans «Un demi-siècle d'environnement entre science, politique et prospective» (Barré et al., 2015).
}

appellent tout particulièrement un type de connaissance proche de celle que ces programmes ont permis de produire, ils sont condamnés à disparaître.

\section{Quelles recherches futures pour la transition écologique et énergétique?}

Dans quelle mesure la conduite des transitions écologiques et énergétiques dans lesquelles nous sommes engagés va-t-elle ou pas renforcer les besoins de recherche pour les politiques publiques et de quelle nature seront ces besoins ? Pour répondre sérieusement à cette question il faudrait, naturellement, entrer dans le contenu concret de ces deux transitions - ce qu'il serait trop long de faire ici. Quelques réflexions générales à partir de la notion - ou plutôt des notions - de transition tiendront lieu de premier éclairage...

\section{Deux conceptions différentes de la notion de transition}

Il n'existe malheureusement pas de définition précise de la transition écologique. Ou plutôt, comme ce fut le cas pour le développement durable, il en existe deux catégories. D'un côté, une définition institutionnelle selon laquelle «la transition écologique» est ce qui se décline dans la Stratégie nationale de transition, dans la loi de transition énergétique ou éventuellement dans les stratégies territoriales ou internationales. Et de l'autre, une série d'approches plus conceptuelles de la notion générale de transition appliquées aux interfaces entre systèmes écologiques et systèmes socio-économiques. C'est de ces dernières dont il sera question ici.

On y distingue deux conceptions assez différentes de la notion de transition ayant chacune des implications politiques et scientifiques bien spécifiques. Une première approche conçoit la transition comme un passage, un entre-deux, une période d'instabilité et de tâtonnements entre deux phases de stabilité. Des mutations, subies ou pas, se produisent dont on apprécie mal les conséquences et la durée, avec à la fois des opportunités nouvelles et des risques, et des possibilités de ruptures brutales : c'est l'entrecroisement de changements multiples en période de fortes tempêtes et incertitudes. Dans une seconde conception, la transition est plutôt un chemin ou une mise en mouvement vers un changement désiré - avec un horizon qui est défini a priori (Chabot, 2015). Plus que l'incertitude ou le risque, ce qui est alors important, c'est la définition des objectifs, le choix des chemins et les conditions pour les atteindre dans les contraintes de temps définies. Dans le cas de la transition écologique, on voit bien que les deux approches sont indispensables: d'un côté, les urgences et les limites ou irréversibilités écologiques font du temps de l'action et du volontarisme 
des dimensions majeures ${ }^{10}$; mais chacun sait aussi que ni la nature, ni la société, ni mêmes les techniques ne sont totalement maîtrisables et qu'il faut aussi faire la part des incertitudes et de l'imprévisible dans un monde où les mutations s'accélèrent.

\section{Quelles conséquences en termes de recherches pour les politiques publiques?}

On devine très bien, de manière très schématique, le type de recherches que peuvent appeler ces deux conceptions :

- la transition comme phase de mutation instable appelle plutôt des travaux historiques sur les transitions de longue période; l'observation et l'anticipation des mutations en cours ou prévisibles ; une analyse de leurs conséquences positives ou négatives (par exemple: conséquences du changement climatique); une évaluation des limites, effets de seuil et risques de ruptures (à la fois sociales, territoriales ou écologiques); des recherches sur les vulnérabilités et les capacités d'adaptation ou de résilience; et des outils d'action en situation de crise et d'incertitude ;

- la transition comme conduite d'un changement désiré nécessite, elle, plutôt, des recherches utiles à la définition des objectifs; la mise en évidence et la comparaison de chemins de transition possibles; la compréhension des dynamiques, conditions et effets des actions à mettre en œuvre; une explicitation des marges de manœuvre, facteurs limitants et risques de blocages, ou au contraire des incitations efficaces et des opportunités qui peuvent accélérer ou freiner l'innovation et le changement - et, naturellement, des outils d'évaluation des progrès réalisés.

Ces deux approches ont en commun d'une part, la prise en compte des possibles (ce qui nous renvoie à l'innovation, l'analyse des risques et incertitudes, la veille et à la prospective) et, de l'autre, une meilleure appréciation des temporalités (des dynamiques, des inerties, des irréversibilités, qu'elles soient naturelles, socio-économiques ou politiques). Il ne faut pas oublier, en effet, que ce qui compte dans la transition écologique, énergétique ou climatique, ce n'est pas seulement de faire les choses, mais aussi de les faire à temps. Or, la recherche est encore relativement silencieuse sur ces temporalités.

\footnotetext{
${ }^{10}$ Le temps est une dimension essentielle des politiques de l'environnement, avec à la fois de fortes inerties, des irréversibilités, des effets cumulatifs et des délais d'action importants. Voir sur cette dimension majeure le texte de J. Theys, «Le climat, une question de temps», publié dans le numéro spécial de NSS consacré à la Conférence de Paris (NSS, 23, S1, 2015a). Voir aussi Theys, 2015b. Les temps de l'écologie, in Bourg et Papaux (Eds.), Dictionnaire de la pensée écologique; et Theys, 2015c. Environnement, d'un demi-siècle à l'autre, Futuribles, 409.
}

\section{Un besoin majeur de décloisonnement}

Quels que soient les enjeux précis qui seront fixés à cette recherche sur la transition écologique ${ }^{11}$ il est clair, en tout état de cause, qu'une des conditions majeures pour qu'elle puisse être utile aux politiques publiques et à l'action collective est qu'elle réussisse à surmonter l'obstacle d'un cloisonnement excessif des approches et des disciplines - toutes ces rationalités en silo qu'illustre l'image de "l'éléphant blanc» ${ }^{12}$. On sait très bien, en effet, que pour réussir la transition, aucune solution unique ne suffira et qu'il faudra combiner les actions, les connaissances, les volontés de mobilisation, et l'intelligence de tous à toutes les échelles. On sait aussi qu'il faudra décloisonner l'environnement ou l'énergie pour s'intéresser aux systèmes productifs, aux modes de vie, à l'aménagement du territoire, à la géopolitique, etc. On sait enfin, mais c'est une évidence, qu'il faudra articuler les disciplines. Or, ces liens indispensables ne se font pas ou se font mal. Ce qui domine, au contraire, ce sont les effets de coupure : par exemple entre sciences « dures » et sciences sociales, innovation technique et innovation sociale, approches sectorielles et territoriales, environnement et développement économique ; mais aussi entre prospective et recherche, modélisation et débat public, recherche et société... Ce devrait être sans doute l'un des objectifs majeurs de la recherche future pour les politiques publiques de construire ou renforcer ces passerelles entre mondes séparés, sans lesquelles il est probable que la transition écologique sera un échec. Ce qui devrait conduire à privilégier des recherches situées aux interfaces (par exemple sur le mimétisme écologique, les modèles conviviaux facilitant le débat public ${ }^{13}$ ou l'innovation ouverte...) et les approches systémiques (outils de simulation des transitions des grands

\footnotetext{
${ }^{11}$ Il faudrait sortir de cette abstraction et décliner ce que cela signifie concrètement, par exemple, pour la transition énergétique ou vers des villes post-carbone, la gestion des océans ou littoraux, de la biodiversité ou des forêts, la transition alimentaire ou agricole, la résilience territoriale, l'adaptation au changement climatique, l'économie circulaire, etc. Mais comme je l'ai dit, cette abstraction est volontaire: je ne me sens pas légitime pour définir des thèmes prioritaires, et préfère donc m'en tenir à des réflexions générales.

${ }^{12}$ Référence à ces moines bouddhistes aveugles qui ayant rencontré et touché un éléphant blanc racontent qu'il s'agit d'un tronc d'arbre (une des pattes), d'un serpent (la trompe) ou d'un animal marin (la défense) [...] (Theys, 2016).

${ }^{13}$ Référence, notamment, aux exercices de simulation des politiques publiques développés par L'IIASA (International Institute for Applied Systems Analysis) et décrits, en France, par Laurent Mermet. Ils permettent de simuler «en temps réel» des scénarios co-construits par des acteurs en charge de concevoir ou de débattre des politiques publiques, aidés par des modélisateurs.
} 
éco-socio-systèmes: grands bassins hydrographiques, villes, littoraux, espaces ruraux...).

\section{Quelle place future pour la recherche pour les politiques publiques et pour la prospective en phase de transition?}

La simple évocation de tous ces thèmes de recherche donne à elle seule une idée de l'ampleur des besoins de recherche finalisée pour les politiques futures de transition. C'est ce que suggère d'ailleurs la Stratégie nationale pour la transition vers un développement durable $^{14}$. Mais aujourd'hui la question n'est pas seulement celle des futurs objets de recherche (même s'ils restent à déterminer) mais, plus fondamentalement celle du positionnement et de l'existence même de cette recherche pour les politiques publiques dans une autre «Grande transition» - qui est celle du système scientifique lui-même et, au-delà, des formes de connaissance et d'action publique. Face aux changements majeurs qui affectent déjà ou vont affecter ce contexte, quels vont être les atouts ou les faiblesses de la recherche pour les politiques publiques ? Comment va-telle devoir évoluer? Pourra-t-elle surmonter la crise liée à la disparition des programmes incitatifs pilotés par le ministère de l'Environnement? Et finalement saura-telle répondre aux besoins de la transition écologique? Pour aborder, brièvement, ces interrogations, il est bon de commencer par rappeler les atouts de cette recherche face à la transition écologique.

\section{La recherche sur les politiques publiques: chaînon manquant de la recherche sur la transition écologique}

On sait le rôle majeur qu'a joué la recherche fondamentale pour nous alerter sur les défis qui sont ceux de la transition écologique ou climatique dans laquelle nous sommes engagés. On mesure aussi à quel point les recherches industrielles ou appliquées vont être nécessaires pour la mettre en œuvre dans les entreprises ou les territoires. Mais le «chaînon manquant» entre l'alerte et le déploiement de solutions techniques concrètes rend tout aussi indispensable les recherches pour les politiques publiques. Ce serait, en effet, une illusion de penser que les lois récentes ont définitivement répondu à toutes les questions à la fois scientifiques et de mise en œuvre socio-politique que posent ces transitions - qui ne sont aujourd'hui qu'à peine amorcées. Comme

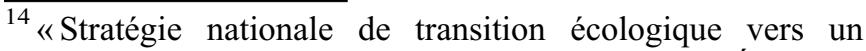
développement durable 2015-2020», ministère de l'Écologie, du Développement durable et de l'Énergie, 2015. La stratégie définit 9 priorités. L'une d'elle porte sur la recherche et à l'organisation de la connaissance.
}

en témoigne la liste, très incomplète, des thèmes évoqués dans la partie précédente, jamais le besoin de ce type de recherches n'a été, en définitive, aussi important. Il y a aussi une adéquation potentielle très forte entre les singularités qui sont celles de ce type de recherche et la nature des processus à développer pour concrétiser la transition écologique. Encore faut-il que ces singularités soient non seulement reconnues, mais aussi sans doute adaptées pour faire face aux besoins futurs.

\section{Des singularités à reconnaître, mais aussi à adapter: le rôle de la prospective et du débat science-société}

De quelles qualités singulières devront faire preuve les recherches pour les politiques publiques nécessaires à la mise en œuvre de la transition écologique? Ces qualités sont en large partie celles qui caractérisaient déjà la recherche incitative ministérielle dont il a été question précédemment: la co-construction des programmes et des thèmes, une situation en amont des recherches appliquées ou techniques, la transversalité et la pluridisciplinarité, l'ouverture à la société et aux acteurs sociaux, l'ancrage dans les territoires et, au total finalement, ce caractère résolument innovant: la prise de risque. Mais devront s'y ajouter d'autres spécificités liées à la transition écologique. Nous les avons déjà évoquées: l'attention aux trajectoires, aux temporalités et aux incertitudes; la reconnaissance de la pluralité des modèles et des possibles; le souci de mettre les connaissances en débat. Et finalement, une plus grande articulation avec la prospective afin d'être en mesure d'anticiper des opportunités ou des risques à long terme, de dessiner des chemins de transition, d'articuler les temporalités ou de faciliter les décloisonnements et le dialogue à trois entre acteurs publics, chercheurs et société $^{15}$. Au-delà des modèles classiques de prévision, la prospective peut être, en effet, à la fois l'outil dont la transition a besoin pour articuler les urgences écologiques à long terme et les urgences politiques à court terme mais aussi un instrument très efficace de mise en débat.

Dialogue à trois incluant la société : il faut y insister. Car au-delà du dialogue, à deux, de co-construction entre les chercheurs et les acteurs publics, une troisième partie prenante est essentielle aussi bien pour la mise en œuvre de la transition écologique que pour le futur de la recherche pour les politiques publiques : la société civile

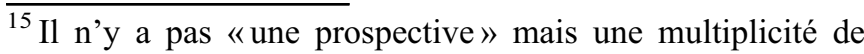
formes ayant des finalités et des fonctions très différentes. Sur ces différences : voir l'interview de J. Theys cité en note ${ }^{7}$ ainsi que Theys $(2015 \mathrm{~d})$ «Entre science et politique, nature et démocratie, la prospective de l'environnement écartelée», également dans l'ouvrage «Un demi-siècle d'environnement entre science, politique et prospective» (Barré et al., 2015).
} 
au sens large du terme, acteurs économiques et locaux compris. Ce qui est, en effet, également en jeu derrière le développement de la recherche pour les politiques publiques et la transition écologique c'est une double reconfiguration à la fois des rapports entre politique et société et entre science et société.

C'est ce qu'exprime fort bien, là encore, Jürgen Habermas, dans son ouvrage de 1968, quand il écrit: «l'alternative ne se situe pas entre un pouvoir politique qui, passant par-dessus la tête de l'opinion et la liberté scientifique, absorberait toutes les ressources de la science et un État coupé de l'information scientifique. Le vrai problème est plutôt de savoir si une fois atteint un niveau de connaissance, on se contente de le mettre à disposition des hommes occupés à des manipulations techniques ou si l'on veut que ce soit des citoyens communiquant entre eux qui en reprennent possession dans leur langage propre, en relation avec leurs pratiques quotidiennes ». Dans les années 1990, la recherche incitative avait été à l'avantgarde de cette ouverture vers la société et les parties prenantes. Avec la transition écologique, cette capacité d'ouverture peut devenir un atout majeur pour la recherche pour les politiques publiques pour peu qu'elle réussisse en partie à se transformer en "recherche pour l'action collective ${ }^{16}$. Encore faut-il, naturellement, que cette autre singularité soit, elle aussi, reconnue.

\section{Un atout dans la transition actuelle des formes de connaissances et d'action publique}

Au-delà des besoins liés à la transition écologique, l'avenir des recherches pour les politiques publiques ne peut pas être pensé indépendamment de la révolution qui est en train de transformer l'ensemble du contexte dans lequel elles se situent. La transition actuelle n'est, en effet, pas seulement écologique, énergétique ou climatique. Elle est globale et touche également les politiques publiques et le monde scientifique dans leurs rapports avec la société - et, au-delà, les formes de connaissances, d'engagement et même de compétences. Dans des sociétés des « sociétés de la connaissance» plus ouvertes, les décisions technocratiques et les expertises trop formelles, basées sur des rationalités trop étroites sont moins acceptées. Les politiques centralisées laissent place à une action collective dans laquelle les questions de compréhension et de mise en débat des décisions

\footnotetext{
${ }^{16}$ Avec le développement de nouvelles formes de gouvernance, on est passé dans les années 90 des "politiques publiques» (pilotées principalement par l'État ou les responsables publics locaux) à l'«action publique» (dans laquelle la société civile et les acteurs privés participent directement à la mise en œuvre d'actions communes pour lesquelles les institutions ne jouent plus qu'un rôle de régulateur ou d'animateur).
}

deviennent centrales. Des tensions traversent les administrations sur ce que doivent être leurs fonctions principales: juridique et régalienne ou stratégique et d'expertise? d'animation indirecte et d'action pédagogique ou de mise en œuvre technique ? Il en est de même dans la communauté scientifique où l'éthique d'autonomie entre en concurrence avec le désir d'engagement social des chercheurs. Et, dans le même temps, les technologies de l'information redistribuent toutes les cartes de la connaissance et brouillent les frontières du savoir. On est dans une phase de recomposition très vaste, qui va à terme transformer en profondeur la production, la demande et la distribution des connaissances, comme la façon d'orienter et de mener les politiques publiques.

Une hypothèse pessimiste serait de craindre que ces évolutions ne conduisent à la fois à une fragmentation extrême de l'action collective, et à une dévalorisation de tout effort d'investissement dans l'information et la recherche. Certains évoquent même pour un demain proche une « ubérisation de l'État» et «de la recherche». Dans ce scénario, la recherche pour les politiques publiques n'aurait plus aucune utilité puisque tout serait désormais directement accessible à coût réduit sur les moteurs de recherche. Mais ce n'est qu'un des avenirs possibles. On peut aussi imaginer une société de la connaissance dans laquelle, au contraire, l'action devra être de plus en plus conditionnée par la construction et le partage de savoirs à la fois approfondis et ouverts. Si elle réussit à préserver sa singularité, la recherche pour les politiques publiques -ou, plus exactement donc, pour l'action collective - aura alors tous les atouts pour s'inscrire avec succès dans cette mutation. Plus ouverte, anticipatrice, pluridisciplinaire et socialement utile, elle pourrait même devenir un modèle pour les autres formes de recherche ou d'expertise. Parier sur son développement, c'est donc parier sur la modernisation de la recherche, mais aussi sur celle des politiques publiques. C'est un atout qui vient s'ajouter à sa bonne adéquation aux besoins futurs de la transition écologique.

\section{Le paradoxe d'une disparition}

Encore faut-il que ces atouts soient reconnus à leur juste mesure et surtout que le développement de ce type de recherche soit assuré, ce qui n'est pas le cas. On est, en effet, aujourd'hui devant le paradoxe d'une disparition de la recherche incitative au sein du ministère en charge de l'environnement au moment où des évaluations mettent enfin en évidence ses qualités et apports spécifiques $^{17}$.

\footnotetext{
${ }^{17}$ Voir les sources citées en note ${ }^{6}$ ainsi que l'ouvrage «Un demi-siècle d'environnement entre science, politique et prospective» (Barré et al., 2015).
} 
Naturellement, cela ne veut pas dire qu'en dehors de ces programmes incitatifs, il n'existe pas de ressources scientifiques qui pourront venir en appui aux politiques publiques. Sur les thèmes qui sont ceux de la transition écologique et énergétique ou de la prise en compte des biens communs, on constate depuis quelques années une forte mobilisation du système scientifique français $-\mathrm{y}$ compris de l'ANR - aussi bien en recherche fondamentale qu'en recherche technique ou appliquée. L'appui aux politiques publiques se développe aussi dans les organismes proches du ministère - notamment à l'ADEME ${ }^{18}$, à l'ANSES $^{19}$, à l'IFSTTAR ${ }^{20}$, à l'IRSTEA ${ }^{21}$ - parmi beaucoup d'autres... Mais, après la disparition de la recherche incitative ministérielle (entraînant par ricochet l'extrême affaiblissement de la prospective en son sein) ${ }^{22}$, trop peu de programmes correspondent au modèle de coconstruction ouvert, pluridisciplinaire et prospectifévoqué ici. Les travaux faits dans beaucoup d'universités ou de grands organismes se situent plutôt en aval ou en amont des politiques publiques; et, comme Rémi Barré l'a montré récemment, la recherche française, trop cloisonnée, n'a pas encore réellement fait son adaptation aux enjeux qui sont ceux du développement durable (Barré, 2015). Certes la prospective se développe dans les organismes scientifiques $^{23}$, mais ses moyens restent toujours extrêmement limités. Et le réseau scientifique proche du ministère, organisé par grands domaines séparés, a tendance à limiter ses travaux en appui des politiques publiques à des problèmes de mise en œuvre situés en aval. On peut craindre, dans ces conditions que la disparition de la recherche incitative, n'accroisse encore plus la coupure culturelle -qui reste malheureusement encore très grande - entre le monde de la recherche et celui de l'action publique, y compris au sein du ministère.

Ce n'est pas le lieu ici de faire des hypothèses sur les voies possibles pour surmonter ce paradoxe. Cela dit, on peut être optimiste, car la recherche pour les politiques

\footnotetext{
${ }^{18}$ Agence de l'environnement et de la maîtrise de l'énergie

${ }^{19}$ Agence nationale de sécurité sanitaire de l'alimentation, de l'environnement et du travail.

${ }^{20}$ Institut français des sciences et technologies des transports, de l'aménagement et des réseaux.

${ }^{21}$ Institut national de recherche en sciences et technologies pour l'environnement et l'agriculture.

${ }^{22} \mathrm{Au}$ sein du ministère de l'Environnement, la prospective et la recherche incitative ont été historiquement très liées, la première étant financée, pour l'essentiel, par la seconde. La disparition de la recherche incitative a donc considérablement réduit -à partir de 2011- les moyens de la Mission prospective, créée en 2007 au moment de la fusion équipement-environnement.

${ }^{23} \mathrm{Ce}$ dont témoignent les nombreuses activités actuelles du réseau PROSPER qui réunit les responsables de la prospective des grands organismes et agences de recherche -réseau créé depuis le milieu des années 2000.
}

publiques ne peut raisonnablement disparaître et a devant elle - quel qu'il soit - un avenir. Elle correspond aux besoins qui sont ceux de la transition écologique et s'inscrit dans le sens de l'histoire. On a une bonne idée de ce vers quoi nous devons aller. Reste à trouver le bon chemin pour concrétiser cette préoccupation commune. Mais il y a urgence, car la nature, le climat, la société n'attendront pas.

\section{Références}

Bain P., Maujean S., Theys J., 2008. Agora 2020, Vivre, habiter, se déplacer en 2020, quelles priorités de recherche? $M E D D A D$, http://side.developpement-durable.gouv.fr/ EXPLOITATION/DRCENT/doc/IFD/IFD REF DOC 0503642/agora-2020-vivre-habiter-se-deplacer-en2020-quelles-priorites-de-recherche.

Barré R., 2015. Développement durable et système français de recherche : vraies difficultés et faux-semblants, in Barré R., Lavoux T., Piveteau V. (Eds.), Un demi-siècle d'environnement entre science et politique, Versailles, Quae, 175188.

Barré R., Lavoux T., Piveteau V., 2015. D’un demi-siècle à l'autre : 50 questions à J. Theys, in Barré R., Lavoux T., Piveteau V. (Eds.), Un demi-siècle d'environnement entre science, politique et prospective, Versailles, Quae, 15-105.

Bourg D., Papaux A., 2015. Dictionnaire de la pensée écologique, Paris, PUF.

Callon M., Rip A., 1992. Humains, non-humains : morale d'une coexistence, in Theys J., Kalaora B. (Eds.), La Terre outragée. Les experts sont formels !, Paris, Éditions Autrement, 140-156.

Chabot P., 2015. L'âge des transitions, Paris, PUF.

Charvolin F., 2005. L'invention de l'environnement en France, Paris, La découverte.

Dahan A., Guillemot H., 2015. Les relations entre science et politique dans le régime climatique : à la recherche d'un nouveau modèle d'expertise, Natures Sciences Sociétés, 23, S1, https://nss-journal.org/fr/articles/nss/abs/2015/02/ nss 150014/nss150014.html.

Godard O., 2008. Les relations sciences-société à l'aune du développement durable, in Lesourne J., Randet D. (eds.), $L a$ recherche et l'innovation en France, Futuris 2008, Paris, Odile Jacob, 353-402.

Habermas J., 1973. La technique et la science comme «Idéologie», Paris, Gallimard. Traduit de : Technik und Wissenschaft als"Ideologie”, Suhrkamp Verlag, Auflage, 1968.

Theys J., 2010. Trois conceptions irréductibles de l'environnement, in Coutard O., Lévy J.-P. (Eds.), Écologies Urbaines, Paris, Economica, 15-24.

Theys J., 2015a. Le climat, une question de temps, Natures Sciences Sociétés, 23, S1, 1-2, https://nss-journal.org/fr/ articles/nss/abs/2015/02/nss150012/nss150012.html.

Theys J., 2015b. Les temps de l'écologie, in Bourg D., Papaux A. (Eds.), Dictionnaire de la pensée écologique, Paris, PUF. 
Theys J., 2015c. Environnement, d'un demi-siècle à l'autre, Futuribles, 409, 57-73, https://futuribles.com/fr/revue/409/ environnement-et-climat-dun-demi-siecle-a-lautre/.

Theys J., 2015d. Entre science et politique, nature et démocratie, la prospective de l'environnement écartelée, in Barré R., Lavoux T., Piveteau V. (eds.), Un demi-siècle d'environnement entre science, politique et prospective, Versailles, Quae, 255-270.
Theys J., 2016. Transition énergétique : le syndrome de l'éléphant blanc, Revue Projet, http://revue-projet.com/ articles/2016-01-theys-transition-energetique-le-syn drome-de-l-elephant-blanc/.

Theys J., Kalaora B., 1992. Quand la science réinvente l'environnement, in Theys J., Kalaora B. (Eds.), La Terre outragée. Les experts sont formels !, Paris, Éditions Autrement, 15-49.

Citation de l'article : Theys J., 2017. Prospective et recherche pour les politiques publiques en phase de transition. Nat. Sci. Soc. 25, S84-S92. 